\title{
The Removal of 4-Chlorophenol and Dichloroacetic Acid in Water Using Ti-, Zr- and Ti/Zr-Pillared Bentonites as Photocatalyst
}

\author{
${ }^{1}$ Mohamed Houari, ${ }^{2}$ Mohamed Saidi, ${ }^{1}$ Djamel Tabet, ${ }^{3}$ Pierre Pichat and ${ }^{1}$ Hussein Khalaf \\ ${ }^{1}$ Department of Chemical Engineering, University of Blida, \\ P.O. Box 270-09000 Blida, Algeria ${ }^{2}$ Centre Universitaire, Medea, Algeria \\ ${ }^{3}$ Laboratoire Photocatalyse, catalyze et Environment, CNRS UMR If OS \\ Ecole Centrale de Lyon, 69134 Ecully Cedex, France
}

\begin{abstract}
Heterogeneous photocatalysis could be alternative remediation technology for water since it does not need the addition of any chemicals and it is suitable for treating low concentrations of pollutant. Although the $\mathrm{TiO}_{2}$ Degussa P 25 is most used photocatalyst its photonic efficiency still low and its recovery from water is considered as an awkward process. In this study the effect of zirconium addition to titanium was investigated. Ti/Zr-pillared montmorillonites have been prepared from natural bentonite and characterized by UV-Vis DRS and X-ray diffraction. The photocatalytic activities have been tested for the removal of 4-chlorophenol and dichloroacetic acid in water. The influence of preparation conditions and the calculation method, on these activities has been investigated. It was found that the photocatalytic activities increase by the addition of zirconium in pillorying process and the calculation by Microwaves (MW) improves the photocatalytic activities.
\end{abstract}

Key words: Photocatalytic Activities, Zirconium, Calcinations, Microwaves, Photonic Efficiency

\section{INTRODUCTION}

The need for water reuse on the large scale, particularly in the countries with limited hydric resources, becomes increasingly a reliable option. This is due to the non availability of water at low prices. The halocarbons in particularly Chlorophenols (CP) and Dichloroactic (DCAA) are abundant industrial toxic component as it has been recognized from different polluting sources, like herbicides, pesticides, chemical and solvent manufacturing and the paint industry. The presence of recalcitrant pollutants in aqueous environment is the principal obstacle, which up to date prevents the water recycle. The 4-chlorophenol, characterized by its acute toxicity and low biodegradability ${ }^{[1]}$ and dichloroacetic acid is considered as animal carcinogens with a qualitative target level of $50 \mu \mathrm{g} \mathrm{L}^{-1}$ in drinking water ${ }^{[2]}$. They are not degradable by conventional water treatment, enabling them to go through the wastewater treatment station without being abated.

One of very promising technology, based on the total mineralization of hazardous organic compounds that are hard to degrade is the heterogeneous photocatalytic oxidation. This technology can be an alternative remediation technology and much attention has been paid to its application in the aqueous environment since (i) it does not need the addition of any chemical; (ii) it is suitable for treating water with low concentrations of organic pollutants, (iii) it is not specific and (iv) it can lead to total mineralization of organic compounds.
Indeed in the natural aqueous system a larger part of the purification is caused by sunlight initiating the decomposition of recalcitrant compounds into simpler molecules. The use of semiconductor catalysts promotes the oxidation processes, which occurs on semiconductor surfaces.

This technology combines heterogeneous catalysis with solar energy, which can be of particular interest for sunny countries.

Some $\mathrm{TiO}_{2}$ semiconductors are by far the most active photocatalyst applied for degrading organic pollutants dissolved in gaseous or aqueous phases ${ }^{[3]}$.

As it is well known, when $\mathrm{TiO}_{2}$ is illumined with UV irradiation an electron is promoted from the valence band to the conduction band to give an electron-hole pair.

The photocatalytic oxidation reactions often described for irradiated titanium dioxide are summarized as follows:

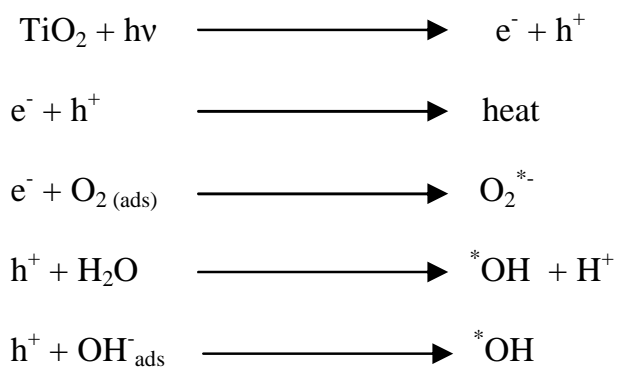

Equation 1 represents the rate of electron-hole Generation in the titanium dioxide. Equation 2

Corresponding Author: Hussein Khalaf, Department of Chemical Engineering, University of Blida, P.O. Box 270-09000, Blida, Algeria 
represents the rate of electron-hole recombination, which occurs within the surface and the volume of the particle. The produced hydroxyl radical, which is known as a very strong oxidant attack the aromatic ring of 4-chlorophenol and the reaction, can lead to total mineralization in the form of $\mathrm{CO}_{2}$ and $\mathrm{H}_{2} \mathrm{O}$.

Presently the most of $\mathrm{TiO}_{2}$ powders have photonic efficiencies for most degradation processes less than $10 \%$. Therefore it becomes evident why novel materials have to be developed and investigated ${ }^{[4]}$.

The improvement of photocatalytic activities can be achieved principally by:

* Decreasing the particle size of photocatalysts down to nanoparticules

* Development and optimization of binary semiconductors nanostructured materials

* Reducing the overall process cost requires avoiding microfiltration by immobilizing the semiconductor catalyst on support.

Several attempts at attaching titanium oxides to a variety of supports, such a glass tube, fiberglass, etc. have been made, as the recovery of micron sized particles from water decontaminated by a $\mathrm{TiO}_{2}$ slurry may be an awkward process. Generally the efficiency of the overall process decreases as the catalysts are immobilized because the illuminated surface area is smaller by several orders of magnitude ${ }^{[5]}$.

The progress realized in the clay pillaring process by various metallic species points to the application of semiconductor-pillared clays as photocatalysts. The intercalated semiconductors in the interlayer spaces of clays, in the form of nanosize pillars could improve its photocatalytic activity. In addition, the high adsorption capacity of clay and its high surface area could to facilitate the retention of the pollutants and their intermediate products of photocatalytic degradation. Finally, pillared clays easily flocculate ${ }^{[6-9]}$. Mixed pillaring by coupling the semiconductors allows enhancing the photonic efficiencies of the final solids and the same time produce large micropores, improving the thermal stability and increasing the surface acidity. Although the mixed pillared smectites has been investigated in catalysis, to our knowledge this the first time where an attempt is made to explore the photocatalytic activity of coupled semiconductor in mixed pillared bentonite as photocatalysts.

The aim of this study was to study, the influence of the addition of another semiconductor (zirconium oxide) and the synthesis conditions on photocatalytic activity of these based bentonite obtained catalysts.

\section{MATERIALS AND METHODS}

Bentonite from Maghnia in Western Algeria has been used as starting material for preparing the photocatalysts. It was firstly purified and harmonized as described in our previous study ${ }^{[6]}$, which we sum up briefly here. The raw bentonite is dispersed in $1 \mathrm{M}$ $\mathrm{NaCl}$ solution in order to have harmonized $\mathrm{Na}$ motmorillonite, then it is separated from the solution and washed several times with distilled water. For separating the fraction of size $<2 \mu \mathrm{m}$, a suspension of 2 wt $\%$ is placed in gradual cylinders for allowing particles $>2 \mu \mathrm{m}$ to settle down. The suspension at the depth of $10 \mathrm{~cm}$ containing only the particles of size $<2$ $\mu \mathrm{m}$ is collected with an Andreason pipette. This operation is repeated several times until the suspension becomes almost transparent at the depth of $10 \mathrm{~cm}$. The particles of a size smaller than $2 \mu \mathrm{m}$ are recovered by centrifugation, washed with distilled water and finally dialyzed to eliminate chloride ions in excess. This fraction was used in the pillaring process. Titanium tetraisopropoxide [ $\mathrm{Ti}\left(\mathrm{C}_{3} \mathrm{H}_{4} \mathrm{O}_{2}\right)_{4}$ ] was used as the precursor of $\mathrm{TiO}_{2}$. Hydrochloric acid was added to it, under vigorous stirring, in order to obtain a $\mathrm{H}^{+} / \mathrm{Ti}$ molar ratio of 4 . To this mixture, distilled water was added and the resulting white sole was peptized further for several hours until a clear solution was obtained. Zirconyl chloride $\left(\mathrm{ZrOCl}_{2}\right)$ was used for preparation of the zirconium pillaring solution.

The intercalation was performed by adding, drop by drop, the pillaring solution to the clay suspension with vigorous stirring. The stirring was then continued for three hours. The solid was separated by centrifugation, rinsed several times with distilled water, dried and finally calcined at various temperatures or by microwave (MW) in a commercial MW oven operating at $2.45 \mathrm{GHz}$ and at a power level up to $300 \mathrm{~W}$ for 15 min. The calcined solids have been characterized by UV-Vis. Spectroscopy using a Perkin-Elmer spectrophotometer lambda 45 equipped with a Labsphere Diffuse Reflectance (DR) accessory.

Tests have been performed in a cylindrical 125 $\mathrm{cm}^{3}$-batch reactor made of borosilicate glass open to air and comprising at its basis an optical Pyrex glass window. The suspension was continuously stirred using a magnetic bar and irradiated by a high-pressure mercury lamp (Philips HPK $125 \mathrm{~W}$ ). An optical filter (cutoff at $340 \mathrm{NM}$ ) and a $2 \mathrm{~cm}$ thick glass cell in which cold water was circulated (to prevent warming of the suspension by infrared irradiation) were interposed between the lamp and the photoreactor (Fig. 1). The radiant power entering the reactor was $445 \mathrm{~mW}$; it was calculated to correspond to $c a .1 .4 \times 10^{18}$ photons per second potentially absorbable by $\mathrm{TiO}_{2}$.

The experiments were carried out with $20 \mathrm{~cm}^{3}$ of solution containing $1.55 \times 10^{-4} \mathrm{~mol} \mathrm{~L}^{-1}(20 \mathrm{ppm})$ of 4chlorophenol or $1.54 \times 10^{-4} \mathrm{~mol} \mathrm{~L}^{-1}$ (20 ppm) of dichloroacetic acid. The powder photocatalyst was added to this aqueous solution in an amount equal to $2.5 \mathrm{~g} \mathrm{~L}^{-1}$. The suspension was stirred in the dark. The concentration, $\mathrm{C}$, of 4-chlorophenol or dichlorlacetic acid decreased slightly and became stable beyond $30 \mathrm{~min}$. 


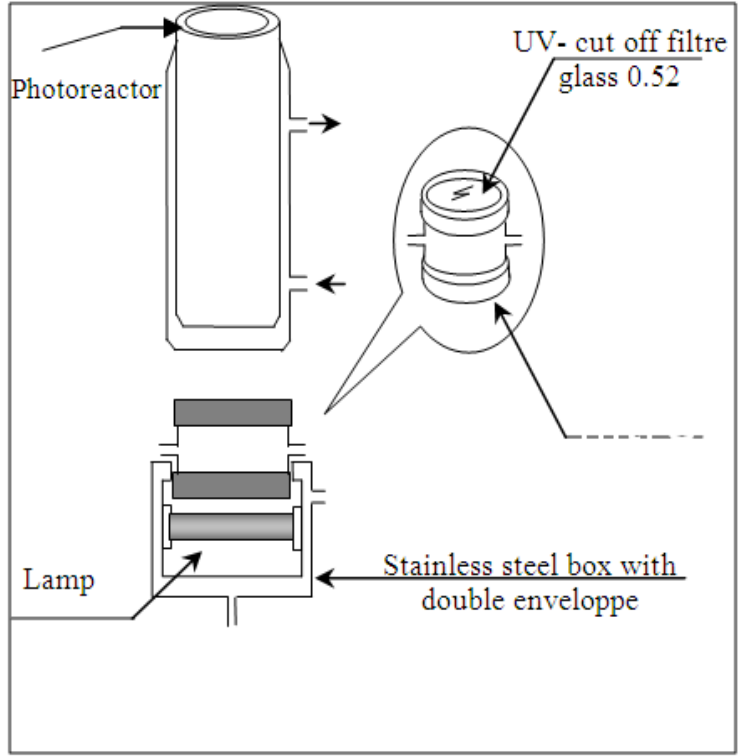

Fig. 1: Schema of the Photoreactor

$\mathrm{C}$ of 4-CP was measured by high performance liquid chromatography using an isocratic pump, a detector adjusted at $226 \mathrm{~nm}$ and a reverse phase $\mathrm{C}_{18}$ column (25 $\mathrm{cm}$ long; $4.5 \mathrm{~mm}$ diameter; $5 \mu \mathrm{m}$ film thickness). The mobile phase consisted of a mixture of water/methanol (40/60), the analysis of dichloroacetic acid was accomplished with HPLC Waters 600 pump equipped with degasser on line, Column SARASEP CAR-H (30 $\mathrm{cm}$ long, $4.6 \mathrm{~mm}$ diameter and $10 \mu \mathrm{m}$ thickness) was used. The detector adjusted at $210 \mu \mathrm{m}$. Aqueous solution of $\mathrm{H}_{2} \mathrm{SO}_{4} 5 \times 10^{-3} \mathrm{Mol} \mathrm{L}^{-1}$ was used as eloquent

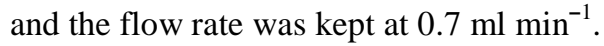

\section{RESULTS AND DISCUSSION}

Solid Characterization: Figure 2 shows the UV-Vis diffuse reflectance spectra of Na-montmorillonite, titanium pillared montmorillonite and titaniumzirconium pillared montmorillonite calcined in conventional oven at $673 \mathrm{~K}$ or by MW.

Compared with the Na-bentonite spectrum, the spectra of pillared clays show an absorption edge at about $400 \mathrm{~nm}$ shifted toward higher wavelength for the mixed pillared solid. The shift of the absorption edge is more pronounced in the case of the Ti/Zr-pillared montmorillonite calcined by $\mathrm{MW}$. This might be due to a change in the size of the $\mathrm{TiO}_{2}$ and $\mathrm{ZrO}_{2}$ particles.

The X-ray diffraction patterns (XRD) of untreated and pillared clay were performed using a Philips model PW 1710 diffractometer and Ni-filtered Co K $\alpha$ radiation. Oriented specimens were prepared by spreading the sample on a glass slide, then exposing it to thermal treatment at $673 \mathrm{~K}$ for 3 hours or irradiated by MW for $15 \mathrm{~min}$.

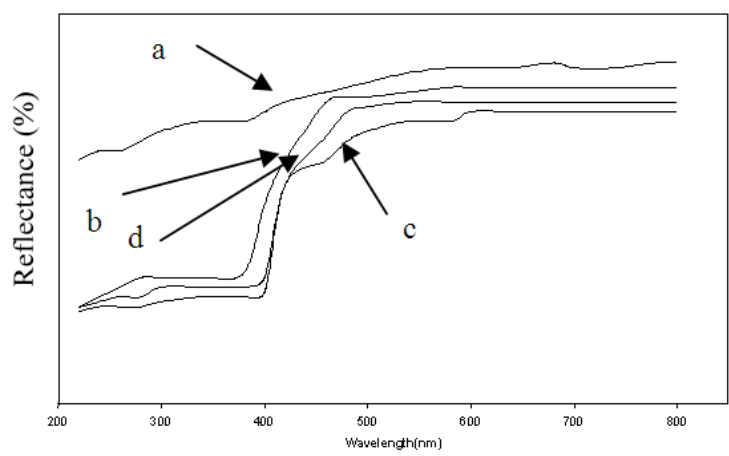

Fig. 2: UV-Vis Diffuse Reflectance Spectra a) Nabentonite; b) Ti-pillared montmorillonite; c) Ti/Zr-pillared Montmorillonite Calcined at $400^{\circ} \mathrm{C} ;$ d) Ti/Zr-pillared montmorillonite Calcined by MW

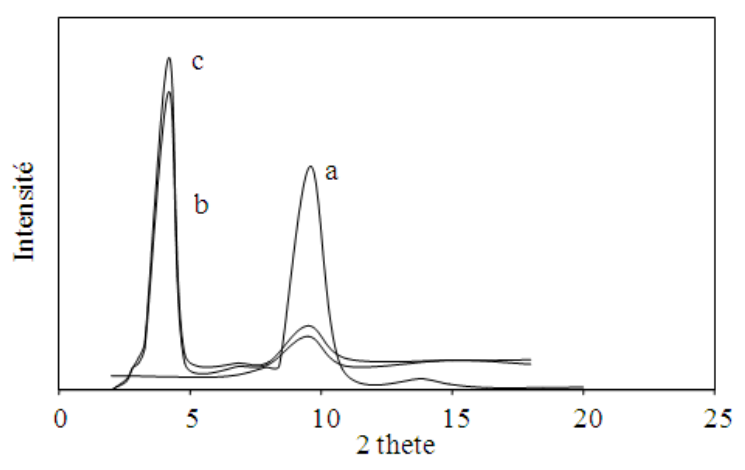

Fig. 3: XRD Patterns of Zirconium and Titanium/zirconium Pillared montmorillonite Prepared at Various Conditions; a- Na-purified Bentonite; b- Ti/Zr-montmorillonite Calcined at $400^{\circ} \mathrm{C}$; c- $\mathrm{Ti} / \mathrm{Zr}$-montmorillonite Calcined by $\mathrm{MW}$

Figure 3 shows XRD patterns in the $2 \theta=20^{\circ}$ range The orientation of the specimens allows the (001) diffraction peak to be more intense. The pattern of untreated Na-montmorillonite shows the (001) peak at $2 \theta=10,2^{\circ}(\mathrm{d}=1,0 \mathrm{~nm})$ typical for montmorillonite, while the pattern of $\mathrm{Ti} / \mathrm{Zr}$-pillared montmorillonites shows a very sharp (001) peak at $2 \theta=4.2^{\circ}(\mathrm{d}=2.4 \mathrm{~nm})$ due to intercalation of polycationc species of titanium and zirconium. This change indicates an important increase in the basal spacing $\mathrm{d}$ (001). These results reveal that the pillared clay samples have good ordered layers structure with insertion of titanium and zircon pillars, which causes an increase in the clay basal spacing.

Photocatalytic Tests: In Fig. 4 are plotted the abatements of 4-chlorophenol concentration as a function of time using Ti-, Zr-pillared or mixed Ti/Zr-pillared samples dried and calcined by various methods, viz. 


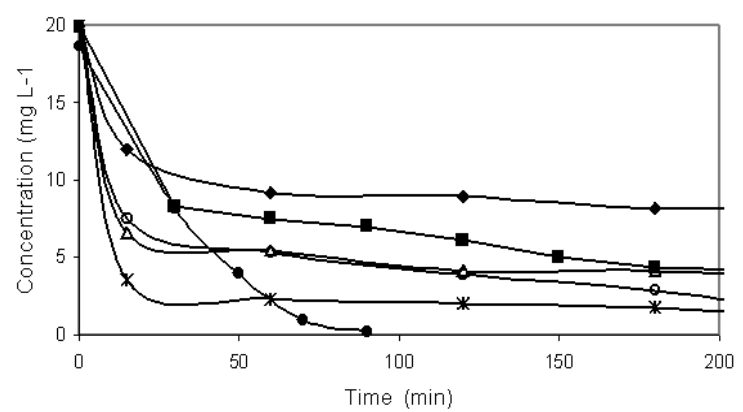

Fig. 4: Concentration Variation with 4-chlorophenol as a Function of Time Various - Zr-pillared Montmorillonite Calcined at $673 \mathrm{~K}$; $\mathbf{m - T i}$ Montmorillonites Calcined at $673 \mathrm{~K}$; $\Delta$ - Ti/Zrpillared Montmorillonite no Calcined; ○-Ti/Zrpillared Montmorillonite Calcined at $673 \mathrm{~K}$ *Ti/Zr-pillared montmorillonite Calcined by Microwave; $\bullet \mathrm{TiO}_{2}$ Degussa, P 25

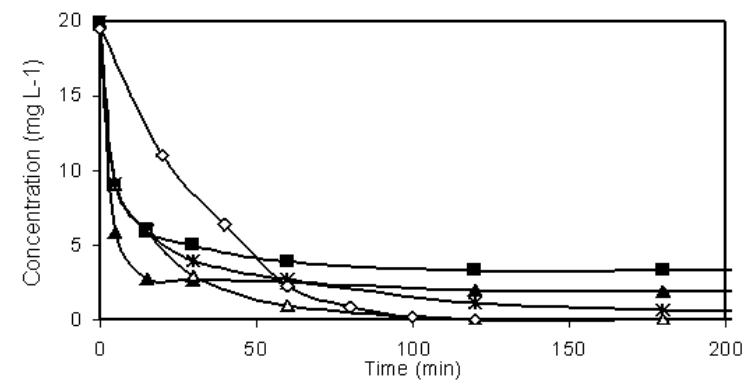

Fig. 5: Concentration Abatement of Dichloroacetic Acid as a Function of Time - $\mathrm{Zr} / \mathrm{Ti}$-pillared Montmorillonite Calcined at $673 \mathrm{~K} ; \boldsymbol{\Delta}-\mathrm{Zr} / \mathrm{Ti}$ pillared Montmorillonites Calcined by MW; *Ti/Zr-pillared Montmorillonite Calcined at 673 $\mathrm{K} ; \Delta$-Ti/Zr-pillared Montmorillonite Calcined by Microwaves; $\diamond-\mathrm{TiO}_{2}$ Degussa, $\mathrm{P} 25$

Calcined at $673 \mathrm{~K}$ in an ordinary oven or calcined by MW. To evaluate the photocatalytic activities of these solids with respect to powder $\mathrm{TiO}_{2}$, the results of one test performed under the same operating conditions, but using $\mathrm{TiO}_{2}$ Degussa P-25, are also plotted. From these curves it can be concluded that the Ti/Zr-pillared clays is more efficient when calcined by MW than the same sample calcined at $673 \mathrm{~K}$ in an ordinary oven. Its photocatalytic activity is even slightly higher than that of $\mathrm{TiO}_{2}$ Degussa P-25 to remove about $90 \%$ of 4chlorophenol. The higher activity of MW calcined sample might result from a higher surface area and perhaps a different pore distribution. It is well known that a solid sample is uniformly heated in the bulk and on the surface when submitted to a MW treatment. Consequently, the MW drying is supposed to facilitate the transport of water molecules toward the surface, unlike a conventional heating where the heat is transferred from the surface of the bulk ${ }^{[10]}$.
Figure 5 presents the results of the removal of DCAA using samples differing by the calcination method and by the order of the intercalation of $\mathrm{Ti}$ and $\mathrm{Zr}$. These results show that the Ti/Zr-pillared montmorillonite is greatly more active than the catalyst obtained by pillaring the montmorillonite by titanium alone, which had a very low photocatalytic activity for DCAA removal. The higher degradation yield with mixed pillared montmorillonite might be explained by an increase in the adsorption capacity due to intercalation by zirconium as it is necessary for DCAA to be in close contact with the photocatalyst to be degraded ${ }^{[11]}$. Furthermore coupling two semiconductors such as $\mathrm{TiO}_{2}$ and $\mathrm{ZrO}_{2}$ might increase the charge separation and accordingly enhance the photonic efficiency. Doping of these semiconductors by the other cation might also favor charge separation ${ }^{[12]}$. On the other hand from the traces in Fig. 5 it can be seen that the photocatalytic activities of $\mathrm{Zr} / \mathrm{Ti}$ pillared bentonite is related to some extent to the preparation method of pillared clays. Samples intercalated first by $\mathrm{Zr}$ polycation and then by titanium show lower activities than the sample intercalated first by titanium and then by zirconium. This may be explained by a lower content of $\mathrm{Zr}_{4}{ }^{+}$cation intercalated in the former case, because the $\mathrm{Zr}^{4+}$ cations cannot replace $\mathrm{Ti}^{4+}$ cations in the interlayer space. The presence of $\mathrm{ZrO}_{2}$ in the nano environment of montmorillonite can affect the acidic properties of the solid. Further research is needed on the basis of these working hypotheses.

\section{CONCLUSION}

On investigating the effect of coupling semiconductors on the photocatalytic activity for the removal of 4-chlorophenol and dichloroacetic acid in the water it was found that mixed pillaring enhance the photocatalytic activity. This enhancement has been shown to depend on the preparation method of these new types of supported photocatalysts. It was more pronounced in the case of DCAA elimination. Further work is needed in particular on the effect of the ratio of the semiconductor on the interaction of $\mathrm{Zr}-\mathrm{Ti}$ in the nano environment of the clay and accordingly on their photocatalitic efficiency.

\section{ACKNOWLEDGEMENT}

Financial support from the "Comité Mixte d'Evaluation et de Prospective de coopération interuniversitaire Franco-algériennne" (C.M.E.P.) within the framework of project 02 MDU 556 is gratefully acknowledged. In particular, this project allowed the photocatalytic activity tests to be carried out in France. 


\section{REFERENCES}

1. Calvo, L., A.F. Mohedano, J.A. Casas, M.A. Gilarranz and J.J. Rodr_ýguez, 2004. Treatment of chlorophenols-bearing wastewater through hydrotreatment using Pd/activated carbon catalysts. Carbon 42: 1377-1381.

2. Liu, Y. and S. Mou, 2004. Determination of bromate and chlorinated haloacetic acids in bottled drinking water with chromatographic methods. Chemosphere, 55: 1253-1258.

3. Pichat, P., 2003. In: Photocatalytic Degradation of Pollutants in Water and Air: Basic Concepts and Applications. In: Tarr, M.A., Chemical Degradation Methods for Wastes and Pollutants: Environmental and Industrial Applications. Marcel Dekker, Inc., New York, Basel, pp: 77-119.

4. Almquist, C. and P. Biswas, 2000. The photooxidation of cyclohexane on titanium dioxide: An investigation of competitive adsorption and its effects on products formation and selectivity. Appl. Catal. A, 214: 259-271.

5. Fernandez-Ibanez, P., S. Malato and O. Enea, 1999. Photochemical reactor for solar decontamination of water. Catalysis Today, 54: 329-339.

6. Khalaf, H., O. Bouras and V. Perrichon, 1997. Synthesis and characterization of Al-pillared and cationic surfactant modified Al-pillared Algerian bentonite. Microporous Materials, 8: 141-150.
7. Ooka, C., S. Akta, O.Y. Ohashi, T. Horiuchi, K. Suzuki, S. Komai, H. Yoshida and T. Hattori, 1999. Crystallization of hydrotermally treated pillars in pillared montmorillonite for improvement of the photocatalytic activity. J. Mater. Chem. 9: 2943-2952.

8. Ding, Z., H.Y. Zhu, G.Q. Lu and P.F. Greenfield, 1999. Photocatalytic properties of titania pillared clays by different qrying methods. J. Colloid and Interface Sci., 209: 193-199.

9. Ooka, C., H. Yoshida, M. Horio, K. Suzuki and T. Hattori, 2003. Absorptive and photocatalytic performance of pillared montmorillonite in degradation as endocrine disruptors having different hydrophobicity. Appl. Catal. B, 41: 313321.

10. Rajeshkumar, S., G.M. Anilkumar, S. Ananthakumar and K.G.K. Warrier, 1998. Role of drying technique on the development of porosity in silica gels. J. Porous Materials, 5: 59-63.

11. Enriquez, R., B. Beaugiraud and P. Pichat, 2004. Mechanistic implications of the effect of $\mathrm{TiO}_{2}$ accessibility in $\mathrm{TiO}_{2}-\mathrm{SiO}_{2}$ coating upon chlorinated organics photocatalytic removal in water. Water Sci. Technol., 49: 147-152.

12. Wang, Y., M.S.W. Liu, M.K. Lu, F.S. Wang, F. Gu, X.Z. Gai, P.X. Cui and J. Pan, 2004. Preparation and photocatalytic properties of $\mathrm{Zr} 4+$ doped $\mathrm{TiO}_{2}$ nanoparticles. J. Mol. Catal. A: Chemical, 215: 137-142. 